\title{
La Salle des produits anglais du Conservatoire des arts et métiers ou la technologie oubliée
}

The Room of the British products in the Conservatoire des arts et métiers or the forgotten history of technology

Marie-Sophie Corcy et Liliane Hilaire-Pérez

\section{(2) OpenEdition}

Journals

\section{Édition électronique}

URL : http://journals.openedition.org/artefact/622

DOI : 10.4000/artefact.622

ISSN : 2606-9245

\section{Éditeur :}

Association Artefact. Techniques histoire et sciences humaines, Presses universitaires du Midi

\section{Édition imprimée}

Date de publication : 15 juin 2017

Pagination : 41-58

ISBN : 978-2-7535-6525-8

ISSN : 2273-0753

\section{Référence électronique}

Marie-Sophie Corcy et Liliane Hilaire-Pérez, " La Salle des produits anglais du Conservatoire des arts et métiers ou la technologie oubliée », Artefact [En ligne], 5 | 2016, mis en ligne le 15 novembre 2017, consulté le 05 mars 2020. URL : http://journals.openedition.org/artefact/622 ; DOI : https://doi.org/ $10.4000 /$ artefact.622 


\section{La Salle des produits anglais du Conservatoire des arts et métiers ou la technologie oubliée}

Marie-Sophie Corcy et Liliane HILAIRE-PÉREZ*

\section{Résumé}

Le Musée des arts et métiers (Paris) conserve une quarantaine d'objets provenant de la mission secrète organisée, en 1819, en Angleterre, pour rapporter au Conservatoire des arts et métiers des échantillons de produits manufacturés. Cette collection, présentée au Conservatoire à l'occasion de la cinquième exposition des produits de l'industrie française, a vite été oubliée. Elle renvoie pourtant aux tentatives mises en œuvre, après le traité de 1815, par les personnalités les plus influentes du monde économique, parmi lesquelles le duc de La Rochefoucauld-Liancourt (1747-1827), pour supporter la rivalité avec l'industrie anglaise.

L'étude des " produits anglais " nous autorise ainsi à proposer une autre histoire de l'industrie anglaise et de ses représentations, au prisme de l'économie du produit, fondée sur l'essor des consommations. La collection des produits anglais révèle autant une image de l'Angleterre irréductible à la seule mécanisation que la pluralité de sens donnés à la technique et à la technologie au Conservatoire des arts et métiers. Cette

*. Marie-Sophie Corcy est ingénieure de recherche et responsable des collections au Musée des arts et métiers-Cnam. Ses travaux portent sur l'innovation dans le domaine de la photographie et des techniques de la communication, mais également sur l'histoire des collections du Musée des arts et métiers, l'histoire des pratiques de gestion et de la muséographie, l'histoire de la médiation et des représentations de l'invention. Elle a co-organisé plusieurs colloques internationaux, notamment « Cabinets de curiosités, collections techniques et musées d'arts et métiers : origines, mutations et usages, des Lumières à la Seconde Guerre mondiale » (2011), qui ont apporté un éclairage nouveau sur les sources et leur analyse en histoire des techniques. Elle a aussi été commissaire des expositions «Eppur si muove » (Mudam-Luxembourg, 2015) et «Laboratoires de l'art » (Musée des arts et métiers, 2016). Contact : [marie-sophie.corcy@cnam.fr.]

Liliane Hilaire-Pérez est professeure d'histoire moderne à l'université Paris Diderot-Paris 7 et directrice d'études à l'EHESS. Elle a publié La pièce et le geste. Artisans, marchands et savoirs techniques à Londres au XVIII siècle (Albin Michel, 2013) et co-édité Histoire des techniques. Mondes, sociétés, cultures (XVI-XVIII siècle) (PUF, Nouvelle Clio, 2016) et L'Europe des sciences et des techniques. Un dialogue des savoirs (XVe-XVIII siècle) (PUR, 2016). Elle co-dirige, avec Stéphane Lembré et Koen Vermeir, la série "Techniques, savoirs, sociétés » aux PUR. Contact : [liliane.perez@wanadoo.fr]. 
étude montre comment la recherche historique sur les collections constitue une voie majeure pour l'histoire de la technologie.

Mots-clés : Conservatoire des arts et métiers (Paris), industrie, produit, révolution industrielle, technique, technologie.

\section{Abstract. The Room of the British products in the Conservatoire des arts et métiers or the forgotten history of technology}

The Musée des arts et métiers (Paris) holds in its possession a collection of approximately forty objects acquired during an 1819 secret mission to Great Britain. The goal of the mission was to procure samples of manufactured goods for the Conservatoire des arts et métiers. The objects collected during this mission were presented at the Conservatoire on the occasion of the fifth Exhibition of the French Industrial Products and then promptly forgotten. Regardless of its subsequent fall into oblivion, the setting up of the British Products Room it was part of the attempts by influent French officials, such as the duke of La Rochefoucauld-Liancourt (17471827), to sustain the competition with British industry in the wake of the Treaty of Paris in 1815.

The study of the "British products » helps further strengthen an alternative narrative of British industrialization and its representations of this period by focusing the product economy and the rise of consumption. This collection of British products is significant for it demonstrates that the image of Britain was not grounded solely in mechanization. Rather, it reveals a plurality of the meanings of "technique » and "technology » that was on display in the Conservatoire des arts et métiers. As such, this article shows that historical research on technical collections constitutes a major path for the history of technology.

Keywords : Conservatoire des arts et métiers (Paris), industrial revolution, industry, product, technique, technology.

En juillet 1817, les membres du conseil de perfectionnement du Conservatoire royal des arts et métiers envisageaient d'organiser une nouvelle exposition des produits de l'industrie française, "une des causes les plus puissantes de l'avancement et de perfectionnements des arts industriels par l'émulation et le mouvement général qu'il imprime à toutes les classes de citoyens ${ }^{1} »$. Le Conservatoire fut pressenti pour accueillir cette exposition. Une commission désignée par le conseil de perfectionnement en août 1818, composée de Jean-Bernard Tarbé de Vauxclairs (1767-1842), viceprésident de l'École des Ponts et Chaussées, de Gérard Joseph Christian (1778-1832), directeur du Conservatoire des arts et métiers, et de Guillaume Louis Ternaux (1763-1833), puissant manufac- 
turier entré en politique au nom de la défense des intérêts industriels français, fut chargée de la définition du projet. Tarbé rapporta au mois d'octobre les travaux de la commission qui avait étudié, outre la périodicité et le coût de l'événement, la question de son emplacement, à Paris. L'esplanade des Invalides, la place Royale, la place Louis XV, le palais de la Bourse (alors en construction) ou bien le palais du Louvre pouvaient accueillir les exposants. Il précisait cependant que le Louvre constituait le « seul emplacement convenable, dont on puisse disposer aujourd'hui » et proposait, " ainsi qu'on l'avait fait pour une exposition précédente, d'établir dans la cour quatre corps de portiques assez vastes pour contenir toute l'exposition ${ }^{2}$ ». L'exposition eut lieu au Louvre mais l'ordonnance royale, signée le 13 janvier $1819^{3}$, prévoyait, à l'article 7, qu' « un échantillon de chacune des productions désignées par le jury, sera déposé au Conservatoire des arts et métiers, avec une inscription particulière qui rappellera le nom du manufacturier ou du fabriquant [sic] qui en sera l'auteur ». Dès 1798, François de Neufchâteau (1750-1828), ministre de l'Intérieur, instaurant la première exposition des produits de l'industrie, avait envisagé une installation permanente au Conservatoire des arts et métiers, tout juste fondét.

Si ces projets n'aboutirent pas, au Conservatoire des arts et métiers, un prolongement de ces expositions eut plus de succès, du moins temporairement. En 1819, alors que Jean-Antoine Chaptal (1756-1832), industriel et chimiste sous l'Ancien Régime et la Révolution, ministre de l'Intérieur de Napoléon Bonaparte (1801-1804), revenait à la vie publique, les membres du conseil de perfectionnement que présidait le duc de La Rochefoucauld-Liancourt (17471827), soulevèrent la question de la présentation des produits étrangers, qui fut bientôt approuvée par le comte de Caze, ministre de l'Intérieur. Le conseil décida de présenter des produits venus de l'étranger, et notamment d'Angleterre, dans une salle du Conservatoire ouverte aux industriels et fabricants français ${ }^{5}$.

Si, en 1798, il s'agissait pour François de Neufchâteau de célébrer le tiers-état, producteur des richesses nationales, d'honorer les artistes et de réhabiliter les arts mécaniques, régénérés par la Révolution, dès la deuxième exposition, en 1801, l'enjeu avait évolué : le but était cette fois de sélectionner les meilleurs fabricants capables de rivaliser avec l'Angleterre. L'exposition se muait en instrument de guerre commerciale avec l'Angleterre ${ }^{6}$. En 1819, lors de la cinquième exposition des produits de l'industrie, le climat de concurrence avec l'Angleterre n'a fait que se renforcer. Depuis la paix en 1815, les échanges à travers la Manche ont repris, la concurrence et les comparaisons aussi ${ }^{7}$.

Mais de quels produits et de quelles techniques s'agit-il? Dans la Salle des produits anglais (en réalité britanniques), si les moyens de production de la grande industrie sont représentés, bien des articles expriment plutôt l'habileté, les savoir-faire et l'ingéniosité attachés à la confection de produits, que ce soit en manufacture ou dans le cadre artisanal. Plusieurs raisons peuvent expliquer ce choix. C'est, d'une part, la place ambiguë de l'Angleterre comme " modèle et contre-modèle » industriel et « la critique de la voie anglaise 
de l'industrialisation ${ }^{8}$ ». D'autre part, si rétrospectivement l'Angleterre a été associée au triomphe du machinisme à mesure que les contemporains construisaient le concept de révolution industrielle et le culte de l'inventeur', au début du XIXe siècle, l'économie du produit, smithienne, fondée sur l'essor des consommations et la diversification des gammes d'articles caractérise toujours l'Angleterre $^{10}$.

C'est cette autre histoire de l'industrie anglaise et de ses représentations que nous nous proposons d'analyser. Il s'agit de comprendre le sens donné à cette collecte d'objets et d'échantillons dans le haut-lieu de la culture technique et, par-là, les conceptions que les contemporains se font de l'industrie, un terme encore largement polysémique. Lorsque Chaptal fonde la Société d'encouragement pour l'industrie nationale en 1801 et publie De l'industrie en $1819^{11}$, l'indus- trie désigne, de manière inclusive, la diversité des fabrications et des activités humaines. Cette définition large de l'industrie fonde le concept de technologie comme science des arts et des intentions de fabrication, un sens effacé par la montée de la science industrielle, mais jamais éteint ${ }^{12}$.

Après avoir présenté la mission de collecte de ces produits en 1819, nous évoquerons la perception des techniques anglaises qui se manifeste à travers cette collection, puis le devenir de la Salle des produits anglais. Cet épisode de l'histoire du Conservatoire des arts et métiers, mentionné par Jean Regnault Armonville (1786-1836) en $1825^{13}$, a été très vite oublié. Si l'oubli est une figure classique en histoire des techniques, souvent associée aux échecs, il concerne aussi la culture technique artisanale et l'alternative qu'elle a pu représenter à $l^{\prime}$ industrialisation et au machinisme ${ }^{14}$.

\section{La mission}

Le conseil de perfectionnement du Conservatoire royal des arts et métiers décida de dresser " un état des articles qu'il convient de faire venir de l'Étranger, particulièrement de l'Angleterre, pour servir de terme de comparaison avec nos produits; que ces échantillons seront disposés dans une salle particulière du Conservatoire des arts et métiers à l'époque de l'exposition, où tous les fabricants français pourront les voir avec la plus grande facilité15 ». Gérard Joseph Christian présenta " un état approximatif des divers échantillons des produits de l'industrie étrangère qu'il conviendrait d'acquérir ${ }^{16}{ }^{\prime}$. Le conseil fut chargé de compléter cette proposition. Le véritable instigateur de la mission est le duc de La Rochefoucauld-Liancourt, président du conseil de perfectionnement et fondateur des écoles d'arts et métiers, dans lesquelles est instauré un pont entre la culture technique artisanale et la formation de technicien ${ }^{17}$. Le duc de La Rochefoucauld-Liancourt rédige un " projet d'instruction pour le voyage » en Angleterre qui décrit les modalités de la collecte et en exprime les enjeux. Il propose de "se procurer, par échantillons, [...] les divers produits de l'industrie 
manufacturière anglaise, afin de pouvoir juger, pour chaque branche, et avec les objets mêmes sous les yeux, l'état réel de tout ce qu'elle fournit aujourd'hui et en faire une comparaison exacte avec nos propres produits ${ }^{18} »$. Le contexte est celui de la concurrence avivée entre la France et l'Angleterre depuis la Révolution française ${ }^{19}$.

Pour préparer le voyage, il convient selon lui de répondre à cinq questions qui s'inscrivent pleinement dans une culture commerciale et une économie $\mathrm{du}$ produit - au point de classer les produits par « variété » et " espèce » face au foisonnement et à la différenciation des articles :

« $1^{\circ}$ Parmi les variétés d'une même espèce de produits, quelles sont celles qu'il faut acheter?

$2^{\circ}$ Comment et où faut-il acheter?

$3^{\circ}$ Quels sont les lieux où s'expédient et se consomment chaque genre et chaque espèce de produits?

$4^{\circ}$ Quel est le prix de fabrique pour chaque objet?

$5^{\circ}$ Enfin comment doit-on expédier pour la France les objets qu'on a achetés? »

Une logique commerciale informe aussi les modes d'acquisition des produits : des réseaux marchands doivent être mobilisés et les artisans sont explicitement désignés comme sources d'information. Le duc recommande de s'adresser « à quelque maison de commerce, à quelque courtier, aux artistes et artisans en différents genres près de qui on cherchera à se faire recommander pour connaître quels sont les magasins les mieux approvisionnés, les endroits où il y a le plus de choix, et où on peut le mieux faire en achats ». Ces stratégies sont aussi celles de tout importateur immergé dans une économie commerciale et artisanale. Le paiement des achats se fit par le système des traites (portées à 35000 francs $)^{20}$.

Emmanuel Molard (1774-1829), dit Molard jeune, sous-directeur du Conservatoire et frère de Claude Pierre Molard (1759-1837) le premier administrateur de l'établissement, fut pressenti par le duc pour assumer cette mission ${ }^{21}$. Au-delà de la position institutionnelle de Molard, ce choix était cohérent. Emmanuel Molard avait été impliqué dans le développement des écoles d'arts et métiers qui émanaient du projet novateur d'enseignement technique lancé par La Rochefoucauld-Liancourt. Cette proximité entre les deux hommes facilita l'insertion de Molard. C'est ainsi qu'il fut question de la prise d'un nom d'emprunt pour garantir le succès de cette entreprise et faciliter l'accès aux ateliers; le patronyme de Molard étant " trop connu », il pouvait « devenir un obstacle à [son] admission dans les ateliers ${ }^{22}$ ». Le conseil de perfectionnement n'a pas jugé utile de prendre cette mesure. Le passeport de Molard mentionnait qu'il voyageait en qualité de propriétaire pour des affaires personnelles; ses lettres de recommandation le désignaient seulement « comme un parent de M. Molard connu dans les arts industriels ».

La mission, que Molard estima " très délicate » et " même très difficile $^{23}$ ", dura cinq mois ${ }^{24}$. Les caisses, au nombre de 26 ou 28, adressées au ministre de l'Intérieur et portant «le mot Conservatoire des arts », arrivèrent en juin $1819^{25}$. Elles furent directement 
transportées au Conservatoire ${ }^{26}$ et Molard envoya en mars 1820 le rapport de la mission au ministre de l'Intérieur, dans lequel il mentionnait la liste complète des échantillons et objets acquis en Angleterre, et faisait part d'observations quant aux modalités d'acquisition ${ }^{27}$ :

\section{« [...] j'ai l'honneur de [vous]} transmettre, un rapport raisonné que j'ai fait sur l'ensemble de cette mission; sur les facilités et les obstacles que j'ai rencontrés. J'examine successivement [ ?] les objets les plus importants et je m'arrête sur la fabrication de ceux qui me paraissent supérieurs aux nôtres. J'ai ajouté six feuilles de dessins ${ }^{28}$ qui représentent la manière de construire les grands bâtiments pour manufactures; les fourneaux de cémentation machines employées pour la fabrication des vis à bois; les moulins à fouler les draps, et les laminoirs forgeurs.

Je termine ce rapport par la spécification de vingt et une machines ou mécaniques ${ }^{29}$ que je regarde comme très importantes à avoir au Conservatoire soit en grand, soit en modèle. Les fonds mis à ma disposition ne m'ayant pas permis d'en faire l'acquisition en Angleterre, mais ayant pu m'en procurer des dessins et des descriptions, je propose à votre Excellence d'en ordonner la construction que je pourrai diriger $^{30}$. » 


\section{Les techniques anglaises au prisme de l'économie du produit}

Depuis la Restauration, la concurrence renaît avec l'Angleterre à mesure que les échanges économiques et les flux d'informations ont repris. En même temps, des techniciens britanniques s'installent en France. Le défi face à l'Angleterre n'est plus militaire mais économique et industriel. En lien, s'affirme en France un parti industrialiste, animé par Chaptal, déjà évoqué, et par l'économiste JeanBaptiste Say et l'ingénieur polytechnicien Charles Dupin. Parce qu'ils sont mus par la contestation de l'ordre aristocratique, Say qui publie De l'Angleterre et des Anglais en 1815 et, dans une moindre mesure, Dupin, auteur des Voyages dans la Grande-Bretagne, se font les apologues $\mathrm{du}$ machinisme anglais ${ }^{33}$. L'industrie revêt un sens hautement politique et l'on sait que c'est dans ce milieu que naît l'expression de "révolution industrielle».

En 1819, l'année même de la mission de collecte, Say et Dupin sont promus professeurs au Conservatoire à l'occasion d'un changement important dans les enseignements $\mathrm{s}^{34}$, l'introduction de nouveaux cours, moins centrés sur la démonstration que sur les connaissances théoriques. Dupin est chargé de la mécanique générale, accompagné par Say pour « l'économie industrielle». La création de chaires rompt avec l'enseignement des débuts du Conservatoire promouvant les savoirs pratiques dans la lignée des écoles lancées par La Rochefoucauld-Liancourt.

Les produits collectés en 1819 reflètent la pluralité de sens accordée à l'industrie à un moment où le culte de la grande industrie n'a pas évincé la valeur des savoir-faire et les logiques de l'économie du produit, fondée sur la variété et le renouvellement des articles, en milieu artisanal aussi bien que manufacturier. L'image de l'Angleterre industrielle ne se réduit en rien à la mécanisation de la production (figure 10, cahier couleur).

Ainsi, loin de toute logique industrielle de production en série, dans son « projet d'instruction », La RochefoucauldLiancourt définit des critères de choix des produits pleinement inscrits dans la culture commerciale et consumériste essentielle au sens donné à la qualité au début du $\mathrm{xIX}^{\mathrm{e}}$ siècle ${ }^{35}:$ : $1^{\circ}$ la perfection, le brillant ou le fini de l'exécution; $2^{\circ}$ la commodité de l'usage; $3^{\circ}$ le bon marché; $4^{\circ} 1^{\prime}$ apparence qu'on a donnée à un produit pour flatter le goût quelquefois bizarre des consommateurs auxquels il est destiné. »

Le soin des façons, la finition des articles, la maniabilité et l'adaptation aux désirs des consommateurs, à leurs goûts, notamment par des variations sur l'apparence, sont les caractéristiques majeures de la production de biens en Angleterre depuis le $\mathrm{XvIII}^{\mathrm{e}}$ siècle, et qui font leur succès en Europe, en concurrence avec les produits français, notamment ceux issus de l'artisanat parisien.

Avant d'examiner le détail des marchandises rapportées et leur insertion dans cette économie consumériste, il convient de prendre la mesure du renouvellement historiographique qui permet une telle analyse. 
Grâce aux travaux sur l'histoire de la consommation et de la révolution des produits au $\mathrm{xVIII}^{\mathrm{e}}$ siècle, l'innovation technique ne se confond plus avec celle des gains de productivité, avec le machinisme et l'essor de la grande industrie ${ }^{36}$. La mise à distance de ces récits s'est accompagnée d'un puissant mouvement de relecture de l'industrialisation attentif au rôle de la demande et à l'essor des marchés (et non seulement aux traditionnels facteurs de l'offre), à la complexité des modes de production et à la pluralité des voies de la croissance, incitant à ne pas confondre l'essor économique anglais avec la seule industrialisation.

Une autre image s'est dessinée : celle d'un bouleversement massif de l'organisation du travail, en rien limité à la mécanisation cotonnière, au charbon et à la vapeur, mais affectant en profondeur toutes les couches du monde du travail et revêtant de multiples visages, selon les régions, les secteurs, les marchés. Avant l'extension de la mécanisation, avant l'augmentation des investissements en capital fixe requérant une forte productivité et des productions de masse pour rémunérer les avances, s'est déployé un autre modèle de croissance, une " croissance smithienne ${ }^{37}$ ", fondée sur la variété croissante des produits pour répondre aux besoins de distinction croissants des classes moyennes urbaines, porteuses de la culture des apparences ${ }^{38}$.

Cette croissance, radicalement différente de l'économie industrielle et des productions de masse, repose sur la conjonction de multiples formes d'innovation, dans l'outillage, dans la mécanisation de certaines opérations, dans la création de nouveaux matériaux et de nouvelles compositions, dans le renouvellement accéléré des modèles et les améliorations de qualité, rarement intégrées aux analyses économiques (miniaturisation, maniabilité, portabilité, précision, etc. ${ }^{39}$ ). Ni limité aux gains de productivité, ni cantonné à la seule variation esthétique, ce modèle d'innovation rend inséparables les savoirs mécaniciens de pointe et l'adaptation qualitative à la demande des acheteurs.

Cette conjonction d'invention et de création recouvre pour les contemporains le sens de nouveauté et de modernitée ${ }^{40}$. En Angleterre, les biens de consommation qui allient des formes inédites, des matériaux nouveaux et des procédés de pointe sont dits «new invented " et sont revendiqués dès le début du siècle par les commentateurs, tels Daniel Defoe, comme un trait distinctif de l'identité britannique.

Ce sont de tels produits que conseille de rapporter La Rochefoucauld-Liancourt, à travers les critères de " perfection, brillant, fini ", de " commodité d'usage " (maniabilité), de "bon marché » et $\mathrm{d}^{\prime}$ « apparence ", caractéristiques de cette économie smithienne, source d'une culture technique sophistiquée, effacée de l'historiographie classique de l'industrialisation, mais au cœur de l'intérêt des contemporains. Les échantillons dont il s'agit de faire l'acquisition, concernent avant tout les activités de transformation - le textile (laine, soie, coton, lin, chanvre, crin, chapellerie), les cuirs et peaux, la corne et l'écaille, la papeterie, le colles, les produits chimiques, les articles métallurgiques (fer, acier, cuivre, fer-blanc, tôle vernie, plaqués, plomb) et les biens de consommation (horlogerie, bijouterie, orfèvrerie, instruments de mathématiques, poterie, faïencerie, porcelaine, cristaux en verre ${ }^{41}$ ). 
La collecte d'Emmanuel Molard répond à ces instructions comme l'atteste son rapport. Les techniques industrielles sont peu présentes : sur les 758 articles, se trouvent seulement « six feuilles de dessins qui représentent la manière de construire les grands bâtiments pour manufactures; les fourneaux de cémentation et de fusion de l'acier fondu; les machines employées pour la fabrication des vis à bois; les moulins à fouler les draps, et les laminoirs forgeurs » et " la spécification de 21 machines ou mécaniques » dont Molard «proposait d'[en] ordonner la construction » au Conservatoire. Voilà pour la grande industrie et la mécanisation. Molard rapporte en outre quelques échantillons de métallurgie (fer, acier, plomb, étain ) et des outils (figure 11, cahier couleur).

Le reste de la liste offre une plongée dans l'économie consumériste anglaise. Aux côtés d'échantillons textiles (dentelles, rubans de soie, bas de soie, tricots, châles - peu de cotonnades, produits phare de l'industrialisation), sont listés de multiples articles de quincaillerie, le toyware qui comprend aussi bien des bijoux, des boutons et des boucles, en alliages ou plaqués, que des accessoires personnels (boîtes à tabac, rasoirs), des montres, des instruments d'optique et de mathématiques, des instruments d'agriculture et un large éventail d'articles domestiques (couverts, fontaines à thé, lampes, ornements divers notamment en bronze, pièces pour volets, portes, fenêtres, ainsi que des produits alimentaires, etc.). Dans ce domaine qui porte l'économie consumériste anglaise, les échantillons de Molard épousent avec précision les critères définis par La Rochefoucauld-Liancourt.
En termes de "perfection, brillant, fini ", on trouve dans le rapport de Molard, des " clefs de montre bijouterie fausse ", des " agrafes dorées pour chaînes de col », des " crayons à coulisse imitant l'or ", des boutons dorés et argentés (figure 12, cahier couleur). Les objets répondant à la « commodité d'usage » sont, par exemple, « des nécessaires à barbe composés de lames de rasoir pour chaque jour de la semaine ", des couteaux, des canifs et des fourchettes et nombre d'outils de Sheffield (faux, outils à bois, limes). Les articles « bon marché » recouvrent des salières de Sheffield «imitant l'argent, d'une grande consommation dans le petit peuple ». La liste détaille aussi les chandeliers, les bougeoirs, les plaques de porte dorées, les lampes portatives, les serrures, les compas, les équerres, les échantillons de fer forgé et de fonte, etc. En somme, une panoplie des produits qui font le succès des modes anglaises depuis le milieu du XVIII $^{\mathrm{e}}$ siècle et tiennent leur valeur de leur parfaite adéquation aux usages, aux désirs, aux goûts des consommateurs.

Quelles sont les provenances, quels sont les fournisseurs? Une géographie issue du $\mathrm{XVIII}^{\mathrm{e}}$ siècle filtre dans la liste de Molard : des lainages de Leeds, des poteries du Staffordshire et, pour le toyware, Sheffield, Birmingham, Londres. De Sheffield viennent des cafetières «du plus beau plaqué ", des couteaux, canifs et fourchettes, « des planches d'acier fondu pour lames de couteaux » "fabrique de Hunstmann à Sheffield » - une désignation typique de l'économie du produit, visant à identifier et hiérarchiser une diversité croissante d'articles par leurs producteurs et non par des procédés ${ }^{42}-$ et nombre d'outils. Ce sont les produits 
qui ont fait la renommée de la ville, tel le Sheffield plate, laiton plaqué d'argent, mais aussi les aciers cémentés qui ont permis l'essor de la coutellerie et de l'outillage, c'est-à-dire des objets maniables, précis, adaptés, que l'on regroupe souvent sous l'appellation "toys and tools", des objets héritiers de la culture du geste, qu'il s'agisse d'accessoires personnels, d'instruments ou d'outils, unis dans un même code marchand et esthétique. La firme de Huntsman, issue de Benjamin Huntsman et reprise par son fils William associé à un fabricant de boutons de Sheffield, Robert Asline (Huntsman \& Asline), un " des premiers fabricants de Sheffield » selon Molard, est emblématique de ce secteur. La mise au point du cast steel, acier coulé en lingots, participe des recherches de transposition et de raffinage des matériaux, visant l'homogénéité des matières. Elle est fondée sur la fusion de l'acier de cémentation (recarburé), blister steel, porté à $1500{ }^{\circ} \mathrm{C}^{\circ}$ dans des creusets d'argile enfouis sous $\mathrm{du}$ charbon de terre. Traditionnellement associé à l'invention de Benjamin Hunstman à Handsworth en 1742, développée dans les années 1760 à Attercliff où l'inventeur intègre sur le même site fourneaux de cémentation et de fusion ${ }^{43}$, l'acier au creuset élargit la gamme des aciers des Hunstman. Leur toyware est principalement composé d'articles munis de lames : rasoirs, ciseaux, couteaux, spécialités du polissage de Sheffield et de son environnement rural $^{44}$. Les outils, issus de la sous-traitance dans le Lancashire, sont des limes, des râpes, des burins, des poinçons, des vrilles et des pinces - les "Lancashire tools", typiques de l'orientation proto-industrielle et horlogère de la région. On sait maintenant que la technique de l'acier au creuset est aussi apparue à Londres, dans le contexte de l'essor du toyware, en lien avec l'horlogerie et le laminage de $\mathrm{l}^{\prime}$ or et de l'argent ${ }^{45}$. À Londres comme à Sheffield, le succès du cast steel est porté par la demande consumériste et les besoins des métiers. Toys et tools sont unis par une culture de l'artifice, par la conformation des matières aux gestes, par un matériau " convergent ", adapté à une large gamme d'usages et de fonctions, toute comme le laiton (brass) et la gamme des alliages en vogue depuis le xviII ${ }^{\mathrm{e}}$ siècle.

On retrouve ces «toys and tools » et cette culture technique portée par les marchés consuméristes pour les objets collectés par Molard à Birmingham : des «boutons dorés et argentés qu'un fabricant de Birmingham lui a donnés en échange d'échantillons français » et des lampes portatives, des bougeoirs qui viennent de la manufacture de Boulton et Watt, à Soho. En 1819, Soho désigne pourtant la Soho Foundry de 1795, le haut-lieu de fabrication des machines à vapeur de James Watt (1736-1819), né de son partenariat avec l'industriel Matthew Boulton (1728-1809) et que poursuivent leurs descendants. Mais, pour Molard, Soho renvoie encore à la manufacture de quincaillerie lancée par Boulton en 176746, où le toyware a permis l'acquisition de savoir-faire dans les alliages et les techniques de précision, cruciaux pour la fabrication de machines à vapeur. Comme le remarquait de longue date Eric Robinson, la production de biens de consommation a préparé celle de biens d'équipements $^{47}$.

Il faudrait aussi souligner la place importante de Londres dans la liste de 
Molard, et la variété des produits, signe que les grandes villes de province n'ont pas encore vaincu la capitale, ce que confirment les relectures de la révolution industrielle, réhabilitant le dynamisme de structures artisanales, liées par des chaînes de sous-traitance qui autorisent la production en nombre d'articles déclinés en modèles et sans cesse renouvelés ${ }^{48}$.

L'Angleterre de Molard est l'Angleterre smithienne, celle des produits et de la consommation, celle de l'économie de la variété et de la qualité, celle des gammes d'articles soignés, brillants et bon marché, portés par un niveau élevé d'habileté artisanale (figures 13 et 14, cahier couleur).

Ce n'est pas un monde passéiste : Molard liste des articles qui ont fait l'objet de patents (brevets), comme les serrures sur le modèle de Bramah « dont le brevet est expiré " (figure 15, cahier couleur). Aux côtés des innovations-produits, la mécanisation trouve sa place : à propos de "pièces de forge pour couteaux et canifs », Molard note qu' $^{\prime}$ " on voit que toutes les pièces d'acier ou de fer qui composent un couteau sont forgées à l'étampe ", c'est-à-dire aux presses à estamper, un outillage accessible aux petits entrepreneurs et qui fait le succès des articles de Birmingham depuis le $\mathrm{XVIII}^{\mathrm{e}}$ siècle. À propos de lames de ciseaux, il ajoute : « À Sheffield, toutes sont forgées aux petites forges et avec du coke ", signe de la vitalité des entreprises de taille modeste dans la métallurgie de transformation qui fait encore la réputation de l'Angleterre. Enfin, au sujet des outils de Sheffield, il précise $q^{\prime}{ }^{\prime}$ « on en fait une exportation considérable, néanmoins ils n'ont pas de moyens économiques de fabrication; la bonne qualité de ces outils vient des bonnes matières qu'on emploie et de la grande division de travail, d'un émoulage facile au moyen des machines à vapeur qui mettent les meules en mouvement ". En 1819, le regard sur l'industrie anglaise n'est en rien figé dans une apologie du machinisme : la qualité des matériaux, la division du travail - typique du modèle smithien - et les machines à vapeur cohabitent, définissant un mode de production hybride.

Au-delà de la question de savoir s'il s'agit d'une image fidèle ou non de l'industrie anglaise, ce qui importe est le sens donné à l'industrie : à travers cette économie du produit, s'exprime une vision inclusive de l'industrie, comprenant autant les produits finis, les objets de goût que les semi-produits, les matériaux et les procédés (ceux qui concourent aux jeux des apparences et ceux qui permettent aussi l'abaissement des coûts de revient, du simple estampage aux machines à vapeur), mais aussi l'organisation du travail. Une telle approche, qui atteste la pluralité des mondes industriels au début $\mathrm{du}$ XIX ${ }^{\mathrm{e}}$ siècle, résonne pleinement avec la conception ambivalente de Gérard Joseph Christian dans son Plan de technonomie en $1819^{49}$. Christian définit l'enjeu théorique de sa technonomie comme l'étude du choix technique "parmi la multitude des moyens », selon que l'on cherche « la qualité ou la perfection des résultats » ou bien « l'économie de l'opération » - une dualité entre l'exigence de qualité et celle de productivité. La technonomie de Christian se tient à la croisée d'une économie du produit, qualitative, fonctionnaliste, et de la science industrielle, quantitative, source de la rationalisation du travail sur le long terme ${ }^{50}$. 
C'est aussi cette ambiguïté qui sous-tend la mission de Molard, lequel se montre probablement plus réceptif encore aux techniques artisanales et aux logiques de qualité. C'est ce qui fait l'originalité de la Salle des produits anglais mais aussi sa fragilité dans l'institution.

\section{La Salle des produits anglais}

Christian fut sollicité dès le débarquement des caisses par le bureau des arts et manufactures pour prendre " toutes les dispositions qui peuvent être nécessaires » à la présentation des produits, soit « à faire disposer sans délai une des salles de l'Établissement, de manière que les produits anglais y soient commodément placés, distribués convenablement, et que le public soit facilement admis à les voir, à les examiner, à en prendre connaissance $^{51}$ ». La « salle des produits anglais » fut équipée d'armoires et de grillages pour recevoir les produits et accueillir le public le temps de l'exposition des produits de l'industrie ${ }^{52}$. Nous ignorons quelle réception en a été faite par les industriels français.

Un épisode suggère néanmoins l'intérêt des entrepreneurs pour les objets rapportés, celui des caisses d'outils de menuiserie qu'Emmanuel Molard fut chargé par le comte de Caze d'acquérir, pour le gouvernement. Quatre coffres d'outils furent achetés à Sheffield, auprès de Samuel Smith ${ }^{53}$. Molard écrit au comte Siméon, ministre de l'Intérieur, dans une lettre en date du 26 juin 1820, que les coffres d'outils, déposés au Conservatoire, " n'y sont nullement nécessaires comme les échantillons, puisqu'il s'y trouve déjà un parfaitement semblable dans les $1^{\text {ers }}$ échantillons anglais ${ }^{54} »$, ajoutant :
«Monsieur le duc De Caze en me donnant la commission de les faire venir, ne me fit point part de ses intentions et j'ignore s'il en a laissé des traces au ministère mais il est présumable que son projet était de les donner comme modèles aux fabricants de ces sortes de choses qui s'étaient le plus fait remarquer à l'exposition. »

Le bureau des arts et manufactures n'avait pas eu connaissance de cette affaire $^{55}$. Abraham Louis Breguet (17471823) se porta acquéreur de deux des quatre caisses d'outils ${ }^{56}$; son offre répondait selon lui au but du gouvernement "puisque [son] intention [était] d'employer une de cses caisses dans mes ateliers, et de donner l'autre à un chef de fabrique d'ébénisterie [qu'il employait] habituellement ». Christian accueillit favorablement cette proposition, ajoutant « qu'il serait difficile de placer ces outils dans de meilleures mains que dans les siennes ${ }^{57}$ ». Une troisième caisse fut acquise dans les mêmes conditions par Guibal $^{58}$.

Le rapport du duc de La Rochefoucauld ${ }^{59}$, adressé au ministre de $l^{\prime}$ Intérieur en février 1819, préconisait l'attribution annuelle de fonds " pour continuer [des] achats des produits de l'industrie étrangère » à des- 
sein de servir efficacement aux progrès de l'industrie nationale et de permettre au Conservatoire des arts et métiers d'asseoir sa vocation. Cette demande ne fut pas suivie par le ministère. Il faut attendre l'attribution de crédits extraordinaires octroyés par le gouvernement à l'occasion de l'exposition universelle de 1851 pour que les collections s'enrichissent de machines et produits étrangers. Mais alors que devint la Salle des produits anglais?

En 1829, Molard et Claude Pouillet, sous-directeur, furent chargés par le conseil de perfectionnement " de visiter le rond-point de la grande église, les archives, les pièces qui touchent à la galerie de l'horloge, celles qui communiquent à la salle des produits anglais et autres lieux où sont entassés de vieux débris de machines; ils feront un rapport au Conseil sur le parti que l'on peut tirer de ces divers objets ${ }^{60} »$. Pouillet adressa, en 1831, au ministre de l'Intérieur un état des produits manquants dans la collection des produits anglais ${ }^{61}$. Il précisait que la "salle particulière ", dans laquelle elle était disposée, n'était pas ouverte au public. Il ajoutait que la collection se trouvait "dans un grand désordre », que " les divers objets y sont mal arrangés et très mal conservés ", et que bon nombre avait disparu, sans espoir de les retrouver dans les galeries. L'inventaire des collections du Conservatoire des arts et métiers, entrepris par la commission nommée en 1841 par le ministre du Commerce, Laurent Cunin-Gridaine (1778-1859) $)^{62}$, et achevé en 1842, comporte une liste des objets existants et manquants à cette date. Sept cent quarante-huit numéros y sont décrits : produits manufacturés, échantillons.
La Salle des produits anglais trouva une autre destination. En 1849, le conseil de perfectionnement décida qu'elle accueillerait les nouvelles acquisitions et les modèles de retour au Conservatoire ${ }^{63}$. En 1850, le conseil de perfectionnement se prononça sur le devenir de la collection qui y était toujours déposée :

«Sur l'invitation de l'Administrateur, le Conseil se rend dans la galerie dite des Filatures pour y examiner l'état des produits anglais achetés en 1819 pour le Conservatoire, et qui, destinés à servir d'indications à l'industrie manufacturière, n'ont jamais été mis sous les yeux du public.

Le Conseil a reconnu qu'après trente et un ans de séjour dans des locaux plus ou moins mal clos, la plupart des tissus et des objets de quincaillerie sont avariés; que le plus grand nombre des autres produits, tels que faïence, verrerie, etc., sont de formes et de dessins hors d'usage, et inférieurs à ce qui se fabrique aujourd'hui en France.

En conséquence, à l'exception de quelques objets de céramique désignés par M. Ebelmen [Jacques Joseph Ebelmen (1814-1852)], le Conseil décide que le reste de ces produits cessera de faire partie des collections; que tout ce qui pourra être employé dans l'Établissement sera porté à l'inventaire du mobilier; et que le reste sera remis au Domaine ${ }^{64}$. »

La collection, soumise à l'expertise des professeurs du Conservatoire, est intégrée en partie à l'inventaire des col- 
lections dans le contexte de la normalisation des pratiques de gestion, instituée en 1849 par le général Morin (17951880). Les objets préservés acquièrent un statut ${ }^{65}$; ils reçoivent un numéro d'inventaire (inv. 01874 à 02386, soit 512 items) et sont déplacés dans les galeries. Les produits anglais sont disséminés au fil des thématiques affichées dans le catalogue des collections, publié en 1851 sous la direction de Morin. Il ne subsiste plus aujourd'hui que quarantedeux objets, parmi les collections d'horlogerie et $\mathrm{d}$ 'instrumentation scientifique, de verrerie et céramique, d'agriculture, témoins de cette aventure. La collection a en effet été l'objet de nombreuses radiations.

Il est intéressant de constater que la " collection chinoise ${ }^{66}$ ", acquise entre 1846 et 1849 , constitue, en tant que telle, un chapitre du catalogue de $1851^{67}$. Cette collection comportait, à l'image de la collection anglaise, des échantillons, des outils et des produits manufacturés. La collection chinoise faisait

\section{Conclusion}

La mission de 1819 pour équiper le Conservatoire des arts et métiers d'une "Salle des produits anglais ", dans le sillage des expositions des produits de l'industrie et dans le contexte de la rivalité croissante avec l'Angleterre, n'a malheureusement pas connu de suite, malgré le désir de La Rochefoucauld-Liancourt de poursuivre ce dessein. La mise en place $\mathrm{du}$ haut enseignement au Conservatoire a pu s'accompagner d'un moindre intérêt alors partie de l'actualité des activités du Conservatoire, à l'image de la collection anglaise en 1819, même si les enjeux étaient différents. Elle n'apparaît déjà plus en tant que collection particulière dans l'édition de 1859 du catalogue des collections du Conservatoire ${ }^{68}$. CuninGridaine, qui annonçait en 1846 aux membres du conseil de perfectionnement l'arrivée de ces produits, fit part de sa volonté d'organiser une exposition dans les galeries ${ }^{69}$. Le Conseil de perfectionnement estima qu'il ne pouvait répondre à cette demande ${ }^{70}$. Nous ignorons si le projet aboutit. En 1849, le ministre annonçait l'envoi d'échantillons d'outils chinois, transmis par de Montigny, consul de France en Chine ${ }^{71}$. Il signe néanmoins la perpétuation au milieu du XIX ${ }^{e}$ siècle, d'une conception extensive de l'industrie et finalement, de la technique, comme activité humaine, alors qu'en même temps le Conservatoire promeut une autre conception de la technique à travers la promotion des sciences appliquées et du génie industriel.

pour les techniques des structures productives héritières de l'artisanat.

Elle témoigne cependant de la vision quasi-encyclopédique de l'industrie défendue par La RochefoucauldLiancourt comme par Chaptal en 1819 dans De l'Industrie, ou encore LouisSébastien Le Normand dans son Dictionnaire technologique (1822-1835), une entreprise éditoriale à laquelle Molard participe $^{72}$. Le Normand dirige aussi les Annales de l'industrie (1820-1827) 
qui publient les rapports des expositions des produits de l'industrie ${ }^{73}$. En France, se déploie une conception de la technologie comme science des arts bien éloignée de la philosophie des manufactures d'un Andrew Ure qui, dans le Dictionary of Arts, Manufactures, and Mines qu'il publie à partir de $1839^{74}$, se donne pour seul but de décrire la transformation des matières premières, par voie mécanique ou chimique, en des " objets généraux de valeur échangeable " (« general objects of exchangeable value ») - déjà la technology comme science industrielle ${ }^{75}$.

La collection de produits anglais révèle autant une image de l'Angleterre irréductible à la seule mécanisation que la pluralité de sens donnés à la technique et à la technologie au Conservatoire des arts et métiers. La recherche sur les collections constitue ainsi une voie majeure pour l'histoire de la technologie.

\section{Notes}

1. Arch. Cnam, 2AA/1, Conseil de perfectionnement, séance du 10 juillet 1817 .

2. Ibid., séance du 22 octobre 1818.

3. Jean-Gabriel-Victor DE MoLÉON, LouisSébastien Le Normand, Description des expositions des produits de l'industrie française, faites à Paris depuis leur origine jusqu'à celle de 1819 inclusivement; renfermant les noms et les adresses de tous les exposants tant nationaux qu'étrangers (servant d'introduction aux Annales de l'industrie nationale et étrangère), Paris, Bachelier, 1824, p. 13-14.

4. Dominique Margairaz, François de Neufchâteau. Biographie intellectuelle, Paris, Publications de la Sorbonne, 2005, p. 341-342.

5. Arch. Cnam, 2AA/1, Conseil de perfectionnement, séance du 10 janvier 1819 . Voir également Archives nationales [ensuite AN], F/12/4864, La Rochefoucauld, « Rapport de l'inspecteur général du Conservatoire royal des arts et métiers à son Excellence le Ministre de l'Intérieur, sur l'acquisition à faire pour le Conservatoire, des produits de l'industrie étrangère ", 19 février 1819.
6. D. Margairaz, François de Neufchâteau, op. cit., p. 342.

7. Jean-Claude Perrot, « Les effets économiques de la Révolution. Trente années de bilan (1795-1825) ", in Jean-Claude PERRot, Une histoire intellectuelle de l'économie politique (XVII ${ }^{e}-\mathrm{XVIII}{ }^{e}$ siècle), Paris, EHESS, 1992, p. 441-469.

8. François Vatin, « Modèle et contre-modèle anglais de Jean-Baptiste Say à Eugène Buret : révolution industrielle et question sociale (1815-1840) ", in Sylvie Aprile et Fabrice Bensimon (dir.), La France et l'Angleterre au XIX siècle. Échanges, représentations, comparaisons, Paris, Créaphis, 2006, p. 69-88; François Jarrige, Au temps des tueuses de bras. Les bris de machines à l'aube de l'ère industrielle, Rennes, PUR, 2009, p. 543-547.

9. Julien VinCENT, "Cycle ou catastrophe? L'invention de la "révolution industrielle" en Grande-Bretagne, 1884-1914 », in Jean-Philippe Genet et François-Joseph Ruggiu (dir.), Les idées passent-elles la Manche? Savoirs, représentations, pratiques (France-Angleterre, $\mathrm{X}^{e}-\mathrm{XX}^{e}$ siècles), Paris, Presses de la Sorbonne, 2007, p. 235-268; Christine MACLEOD, Heroes of invention. Technology, liberalism and British identity 1750-1914, Cambridge, Cambridge University Press, 2007.

10. Liliane Hilaire-Pérez, La pièce et le geste. Artisans, marchands et savoirs techniques à Londres au XVIII siècle, Paris, Albin Michel, 2013.

11. Louis Bergeron, "Présentation ", in JeanAntoine Chaptal, De l'industrie française (1819), Paris, Imprimerie nationale, 1993, p. 7-49.

12. Jacques Guillerme et Jan Sebestik, « Les commencements de la technologie ", Thalès, 1968, XII, p. 1-72, Documents pour l'histoire des techniques, $\mathrm{n}^{\circ} 14,2007$, p. 49-122; Hélène VérIN, «La technologie : science autonome ou science intermédiaire ", Documents pour l'histoire des techniques, $\mathrm{n}^{\circ} 14,2007$, p. 134-143; Éric ScHATZBERG, " Technik comes to America : changing meanings of technology before 1930 ", Technology and Culture, vol. 47, n 3, 2006, p. 486-512; AndréGeorges HAUdricourT, La technologie, science humaine. Recherche d'histoire et d'ethnologie des techniques, François Sigaut éd., Paris, MSH, 1987.

13. Jean Regnault Armonville, Table analytique des ouvrages qui ont servi à la composition de La clef de l'industrie et des sciences qui s'y rattachent, Paris, $M^{\text {me }}$ Huzard, 1825, p. 4.

14. Ken Alder, Engineering the Revolution. Arms and Enlightenment in France, 1763-1815, Princeton, Princeton University Press, 1997; id., « Innovation and amnesia : engineering rationality and the fate of interchangeable parts manufacturing in France ", Technology and Culture, vol. 38, n 2, 1997, p. 273-311. 
15. Arch. Cnam, 2AA/1, Conseil de perfectionnement, séance du 10 janvier 1819 ; voir également AN, F/12/4864, La Rochefoucauld, " Rapport de l'inspecteur général du Conservatoire royal des arts et métiers à son Excellence le Ministre de l'Intérieur, sur l'acquisition à faire pour le Conservatoire, des produits de l'industrie étrangère », 19 février 1819.

16. Arch. Cnam, 2AA/1, Conseil de perfectionnement, séance du 23 janvier 1819.

17. Charles R. DAY, « Le duc de la RochefoucauldLiancourt, un philanthrope libéral ", Les Cahiers d'histoire du CNAM, $\mathrm{n}^{\circ} 1,1992, \mathrm{p} .45-74$.

18. AN, F/12/4864, La Rochefoucauld, «Projet d'instruction pour le voyage en Angleterre ».

19. J.-Cl. Perrot, « Les effets économiques de la Révolution. Trente années de bilan (1795-1825) », art. cit.

20. Les dépenses, prises sur les fonds d'encouragement des arts et manufactures, se portèrent à 36 759,65 francs, Arch. Cnam, 2AA/1, Conseil de perfectionnement 1817-1848, séance du 21 décembre 1820.

21. AN, F/12/4864, lettre du Bureau des arts et manufactures, $3^{\text {e }}$ division, au ministre de l'Intérieur, 12 mars 1819; lettre du Bureau des arts et manufactures, $3^{\text {e }}$ division, à Emmanuel Molard, 12 mars 1819.

22. Ibid., lettre d'Emmanuel Molard au Bureau des arts et manufactures, $3^{\mathrm{e}}$ division, 20 mars 1819.

23. Ibid., lettre d'Emmanuel Molard au ministre de l'Intérieur, 26 juin 1820.

24. Ibid., envoi d'un rapport au ministre de l'Intérieur, 26 décembre 1820.

25. Ibid., lettre du Bureau des arts et manufactures, $3^{\text {e }}$ division, au directeur général des douanes, 25 juin 1819 .

26. Molard mentionne l'arrivée de caisses supplémentaires dans une lettre adressée au ministre de l'Intérieur, 23 mars 1820; AN, F/12/4864, lettre d'Emmanuel Molard au ministre de l'intérieur, 23 mars 1820.

27. AN, F/12/4864, lettre d'Emmanuel Molard au ministre de l'Intérieur, 23 mars 1820.

28. Nous n'avons pu mettre au jour ces dessins parmi les collections du Conservatoire.

29. Nous n'avons pu mettre au jour cette liste.

30. AN, F/12/4864, lettre d'Emmanuel Molard au ministre de l'Intérieur, 23 mars 1820.

31. Archives du Musée des arts et métiers [ensuite Arch. MAM], $10^{\circ}$ 527, rapport du 30 novembre 1819.

32. Pierre LAmARD, Histoire d'un capital familial au XIX siècle : le capital Japy (1777-1910), Belfort, Société belfortaine d'émulation, 1988.
33. Gareth StedmAn JonEs, «The genesis of the "Industrial Revolution" : Jean-Baptiste Say and the French debate on industrie », dans J.-Ph. GENET et F.-J. RugGiu (dir.), Les idées passent-elles la Manche?, op. cit., p. 211-233; Carole CHRIsTEN et François VATIN (dir.), Charles Dupin (1784-1873). Ingénieur, savant, économiste, pédagogue et parlementaire du Premier au Second Empire, Rennes, PUR, 2009; Liliane Hilaire-PÉrez, « Technique, économie et politique entre la France et l'Angleterre (XVII ${ }^{\mathrm{e}-}$ XIX siècles) ", in Patrice BRET, Irina Gouzévitch, Liliane Hilaire-PÉRez (dir.), Les techniques et la technologie entre la France et l'Angleterre (XVII XIX siècles), Documents pour l'histoire des techniques, $\mathrm{n}^{\circ} 19,2010$, p. 7-27.

34. Robert Fox, «Un enseignement pour une nouvelle ère : le Conservatoire des arts et métiers (1815-1830) », Les Cahiers d'histoire du CNAM, $\mathrm{n}^{\circ} 1$, 1992, p. 75-92.

35. AN, F/12/4864, La Rochefoucauld, « Projet d'instruction pour le voyage en Angleterre ».

36. Patrick O'Brien et Caglar KeYder, Economic Growth in Britain and France, 1780-1914. Two Paths to the Twentieth Century, Londres, Allen and Unwin, 1978; Neil McKendrick, John Brewer, John. H. Plumb (dir.), The birth of a consumer society. The commercialization of Eighteenth-Century England, Londres, Europa Publications, 1982; Maxine BERG The age of manufactures 1700-1820, Londres, Fontana, 1985; Pat Hudson (dir.), Regions and industries. A perspective on the industrial revolution in Britain (1989), Cambridge, CUP, 1993; Patrick VERLEY, L'échelle du monde. Essai sur l'industrialisation de l'Occident, Paris, Gallimard, 1997.

37. P. Verley, L'échelle du monde, op. cit., « L'offre et la demande : croissance "smithienne" et croissance "schumpéterienne" », p. 105-109.

38. Daniel Roche, La culture des apparences. Une histoire du vêtement XVII $-X V I I I^{e}$ siècle, Paris, Fayard, 1989.

39. Maxine BERG, « Product innovation in core consumers industries in eighteenth-century Britain ", in Maxine BERG et Kristine BRULAND (dir.), Technological revolutions in Europe, Cheltenham, Edward Elgar, 1998, p. 138-159; John StYLES, « Manufacturing, consumption and design in eighteenth-century England ", in John Brewer et Roy Porter (dir.), Consumption and the world of goods, Londres, Routledge, 1993, p. 527544.

40. Maxine Berg, Luxury and pleasure in Eighteenth-Century Britain, Oxford, Oxford University Press, 2005, p. 6.

41. AN, F/12/4864, La Rochefoucauld, « Projet d'instruction pour le voyage en Angleterre ». 
42. Jean-Yves Grenier, L'économie d'Ancien Régime. Un monde de l'échange et de l'incertitude, Paris, Albin Michel, 1996; id., "Une économie de l'identification. Juste prix et ordre des marchandises dans l'Ancien Régime ", in Alessandro STANZIANI (dir.), La qualité des produits en France (XVIII $-X X^{e}$ siècles), Paris, Belin, 2003, p. 25-53.

43. Kenneth C. Barraclough, Steel making before Bessemer, vol. 1, Londres, The Metal Society, 1984 (l'introduction de fer forgé et de charbon de bois dans le creuset, et non plus de blister, date de 1800) ; Paul BEDFORD, "Converters and refiners : urban steelmaking sites in Sheffield 1700-1850 », South Yorkshire industrial history Society journal, no 1, 1998, p. 7-19.

44. Victoria BeAUCHAMP et Joan Unwin, The historical archaeology of the Sheffield cutlery and tableware industry, 1750-1900, Stroud, Arcus, 2002 ; Clive Binfield et David Hey (dir.), Mesters to masters : a history of the Company of cutlers in Hallamshire, Oxford, Oxford University Press, 1997.

45. Chris Evans et Alun Withey, « An Enlightenment in steel? Innovation in the steel trades of eighteenth-century Britain ", Technology and Culture, vol. 53, $\mathrm{n}^{\circ}$ 2, 2012, p. 2-29.

46. Peter M. JONES, Industrial Enlightenment : Science, technology and culture in Birmingham and the West Midlands, 1760-1820, Manchester, Manchester University Press, 2009; Tann JENNIFER, " Matthew Boulton - Innovator », in Malcolm Dick, Peter M. Jones et Kenneth QuiCKENDEN (dir.), Matthew Boulton. Enterprising Industrialist of the Enlightenment, 2013, Aldershot, Ashgate, p. 33-49.

47. Eric RobInson, « Eighteenth-century commerce and fashion : Matthew Boulton's marketing techniques », Economic History Review, nº 16, 1963, p. 39-60.

48. Charles Harvey, Edmund D. Green et Penelope J. CorfiEld, « Continuity, change and specialization within metropolitan London : the economy of Westminster, 1750-1820 ", Economic history review, vol. 52, $\mathrm{n}^{\circ} 3$, 1999, p. 469-495; Helen Clifford, Silver in London. The Parker and Wakelin Partnership, 1760-1776, New Haven, Yale University Press, 2004; Giorgio RIELlo, "Strategies and boundaries : subcontracting and the London trades in the long eighteenth century ", Enterprise and Society, vol. 9, $\mathrm{n}^{\circ} 2,2008$, p. 243-280; L. Hilaire-PÉrez, La pièce et le geste, op. cit.

49. Gérard Joseph Christian, Vues sur le système général des opérations industrielles, ou Plan de technonomie, Paris, $\mathrm{M}^{\mathrm{me}}$ Huzard \& $\mathrm{M}^{\text {me }}$ Courcier, 1819.
50. Jan SEBESTIK, « De la technologie à la technonomie : Gérard-Joseph Christian », De la technique à la technologie, Cahiers STS, Paris, CNRS, 1984, p. 56-69; François JARRIGE, « La machine de Christian et les débats sur la suppression du rouissage au début de la Restauration ", Atala, no 10, 2007, p. 47-60 ; L. HilAire-PÉREZ, La pièce et le geste, op. cit.

51. AN, F/12/4864, lettre du Bureau des arts et manufactures, $3^{\text {e }}$ division, à Christian, 25 juin 1819; lettre de Christian au Bureau des arts et manufactures, $3^{\text {e }}$ division, 6 juillet 1819 : Christian n'apportera la « dernière main » aux dispositions qu'il a prises que lorsqu'il aura reçu les caisses.

52. Arch. Cnam, 2AA/1, Conseil de perfectionnement, séance du 20 février 1829 : Christian fait état de la dépense (armoires et grillages) pour rendre publique les salles des Produits anglais, des tours et de l'horlogerie : 2400 francs.

53. AN, F/12/4864, lettre d'Emmanuel Molard au ministre de l'Intérieur, 17 janvier 1820 ; rapport du Bureau des arts et manufactures, $3^{\mathrm{e}}$ division, au ministre de l'Intérieur, 31 janvier 1820; lettre d'Emmanuel Molard au ministre de l'Intérieur, 4 février 1820; lettre du Bureau des arts et manufactures, $3^{\text {e }}$ division, à Emmanuel Molard, 11 février 1820 .

54. AN, F/12/4864, lettre d'Emmanuel Molard au ministre de l'Intérieur, 26 juin 1820.

55. Ibid., rapport du Bureau des arts et manufactures, $3^{\text {e }}$ division, au ministre de l'Intérieur, 31 janvier 1820 .

56. Ibid., lettre d'Abraham Louis Breguet au ministre de l'Intérieur, 26 août 1820; lettre du Bureau des arts et manufactures, $3^{\text {e }}$ division, à Joseph Gérard Christian, 26 août 1820.

57. Ibid., lettre de Joseph Gérard Christian au ministre de l'Intérieur, 7 septembre 1820; lettre du ministre de l'Intérieur à Breguet, 21 septembre 1820 .

58. Arch. MAM, $10^{\circ} 473$, lettre de Guibal au ministre de l'Intérieur, 24 mars 1826; ibid., 10474, lettre du Bureau des arts et manufactures, $3^{\mathrm{e}}$ division, à Guibal, 6 avril 1826.

59. AN, F/12/4864, La Rochefoucauld, «Rapport de l'inspecteur général du Conservatoire royal des arts et métiers à son Excellence le Ministre de l'Intérieur, sur l'acquisition à faire pour le Conservatoire, des produits de l'industrie étrangère », 19 février 1819 : «Le Conseil a pensé que M. Molard, sous-directeur du Conservatoire, était un des hommes les plus propres à se charger de cette mission. "

60. Arch. Cnam, 2AA/1, Conseil de perfectionnement, séance du 5 juin 1829. 
61. AN, F/12/4865, lettre de Pouillet au ministre de l'Intérieur [?], 14 décembre 1831.

62. Arch. Cnam, 2AA/1, Conseil de perfectionnement, séances des 10 et 14 novembre 1841 .

63. Arch. Cnam, 2AA/2, Conseil de perfectionnement, séance du 5 avril 1849 : le conseil de perfectionnement décide « $1^{\circ}$ que tous les modèles qui devront entrer dans les collections seront déposés d'abord dans un local particulier afin d'être examinés avec soins avant d'être introduits dans la collection. $2^{\circ}$ que tous les modèles qui sortiront soit pour réparation soit pour un autre motif seront pareillement déposés dans le même local pour être examinés avant leur réintégration dans les collections. La salle des produits anglais est affectée à ce service, des tables seront déposées à cet effet. ». Voir également séance du 24 avril 1849.

64. Arch. Cnam, 2AA/2, Conseil de perfectionnement, séance du 19 mars 1850. Ces objets, remis au Domaine, n'apparaissent pas a priori dans l'État des objets et modèles à remettre au Domaine, établi en 1850 par les autorités du Conservatoire des arts et métiers, à partir des numéros d'ordre attribués lors de l'inventaire de 1842. La collection anglaise a eu un statut particulier et n'a pas été intégrée dans les galeries. Les numéros d'inventaire attribués à la collection anglaise, entre 1850 et 1853, qui ont été livrés au Domaine sont : en 1859 : inv. 02291 [629] : souliers à talons tournants ; inv. 02378 [733] : charrue écossaise montée en bois ; inv. 02381 : tourmenteur anglais (modèle en grand) ; en 1863 : inv. 02382 [737] : charrue américaine de Cottain, en fer, et semoir anglais pour les graines de prés (AN, F/12/4866).

65. La collection des produits anglais peut être rapprochée des modèles déposés à l'issue d'une demande de brevet. Ces objets n'étaient pas communiqués au public pour des raisons de confidentialité, leur gestion était donc autonome des objets placés dans les galeries à destination du public.

66. Voir dans ce numéro Christiane Demeulenaere-Douyère, « Missions commerciales et collections techniques au XIX ${ }^{\mathrm{e}}$ siècle : l'introuvable "musée chinois" de la mission de Chine ".

67. Ministère de l'Agriculture et du Commerce, Catalogue des collections du Conservatoire des arts et métiers, Paris, Guiraudet et Jouaust, 1851, p. 203204.

68. Il est en de même pour la collection tunisienne, acquise en 1853, présentée en tant que telle dans le catalogue des collections de 1855 .

69. Arch. Cnam, 2AA/1, Conseil de perfectionnement, séance du 24 juin 1846.

70. Ibid., séance du 24 juin 1846, lettre de Laurent Cunin-Gridaine, 20 juin 1846.

71. Arch. Cnam, 2AA/2, Conseil de perfectionnement, séance du 3 novembre 1849, lettre de Victor Ambroise Lanjuinais, 13 octobre 1849.

72. Louis-Sébastien LENORMAND et LouisBenjamin Francceur, Dictionnaire technologique, ou nouveau dictionnaire universel des arts et métiers, et de l'économie industrielle et commerciale, Paris, Thomine \& Fortic, 1822-1835.

73. Joost Mertens, « Technology as a science of the industrial arts : Louis-Sébastien Lenormand (1757-1837) and the popularization of technology ", History and Technology, vol. 18, n 3, 2002, p. 203-231; id., " The Annales de l'industrie (18201827) : a technological laboratory for the industrial modernization of France ", History and technology, vol. 20, n², 2004, p. 135-163.

74. Andrew URE, Dictionary of Arts, manufactures, and mines, containing a clear exposition of their principles and practice, Londres, Longmans Green and Co., 1839.

75. Joost Mertens, « The mere handicrafts : Ure's Dictionary (1839-1853) compared with the Dictionnaire technologique (1822-1835) », in Patrice Bret, Irina Gouzévitch et Liliane Hilaire-Pérez (dir.), Les échanges techniques entre la France et l'Angleterre (XVI ${ }^{e}-\mathrm{XIX} \mathrm{X}^{e}$ siècles). Réseaux, comparaisons, représentations, Documents pour l'histoire des techniques, $\mathrm{n}^{\circ} 19,2010$, p. 277-285. 